\title{
The Promotive Effects of PTHrP on the Union of Femoral Shaft Fracture in a Mouse Model
}

\author{
Z. HUANG AND J. XIAO*
}

Department of Orthopaedics III Gutian Hospital, Fourth Hospital of Wuhan, Wuhan City, 430033, China

\author{
Huang and Xiao: Effects PTHrP on Healing of Femoral Shaft Fracture
}

\begin{abstract}
In order to explore the effects of PTH-related peptide on fracture healing in mice, the intervention effects and mechanism of action of PTH-related peptide on femoral shaft fracture models in mice of different genotypes were analysed to obtain a new approach for promoting the healing fractures clinically. A total of 32 8-week old PTH-related peptide wild-type and PTH-related peptide gene knockout heterozygous (PTH-related peptide +/-) mice were selected as the test system and standard femoral shaft fracture mouse models were constructed by intramedullary wire fixation. After model construction, all mice were randomly divided into the wild type control, the PTH-related peptide $+/-$ control, the wild type treated and the PTH-related peptide +/- treated groups, with 8 mice in each group. Mice in both treated groups were administered with $80 \mu \mathrm{g} / \mathrm{kg} / \mathrm{day}$ of PTH-related peptide (1-34) subcutaneously and the control groups received subcutaneous injections of saline. After 2 weeks, all mice were sacrificed and the fractured right femur of each mouse in each group was taken for X-ray examination and micro-CT scan to observe the union of the fracture. Next, the formed callus tissues were paraffin-embedded and stained to analyse and compare the bone formation changes of osteoblasts in the fracture sites of mice. Compared to the wild-type group, the saline-treated mice in the PTH-related peptide $+/$ - group had clear fracture lines with callus volume and bone mineral density significantly reduced; after PTH-related peptide treatment, the fractures in the PTH-related peptide +/- group were tightly connected and a large number of osseous calluses were formed; in addition, the density of the callus was higher, which was significantly different from those in the control group $(\mathbf{p}<\mathbf{0 . 0 5})$. Compared to the wild-type group, the area of cartilage callus of the saline-treated PTH-related peptide +/- group increased by $97 \%(\mathbf{p}<0.05)$; after PTH-related peptide administration, the total callus area and osseous callus area increased significantly, while the area of cartilage osseous significantly decreased compared to the control group and after PTH-related peptide administration, the number of osteoblasts and the percent positive area of collagen $I$ in mice were significantly increased compared to the control group. In conclusion, endogenous PTH-related peptide deficiency could lead to delayed healing of fracture, which could be compensated by PTH-related peptide administration, thereby accelerating the formation of calluses and promoting fracture healing in mice.
\end{abstract}

Key words: The union of fracture, femoral shaft fracture, PTHrP, gene deletion, porosis

The fracture refers to the destruction of bone continuity and integrity. The union of fracture afterward is a process of the gradual recovery of bone continuity and integrity, which is a long and complicated pathological process and usually takes a long time[1]. The union of fractures is divided into 2 types: the direct union of fracture and the indirect union of the fracture. The direct union of fracture is also called the primary union of fracture; it means that the fracture ends reach the bone connection through direct osteogenesis and bone unit reconstruction. The indirect union of fracture is also called the secondary union of fracture, which includes the hematoma inflammatory organization stage (completed within $2 \mathrm{w}$ after fracture), the original porosis stage (lasted for 4-8 w), and the bone lamella formation and shaping stage (lasted for $8-12 \mathrm{w}$ )[2]. Clinically, the union of fracture is mostly the indirect union of fracture and the overall union time takes about 18 to $20 \mathrm{w}$.

Most patients with fractures have to stay in bed for a long time because of the need to immobilize their limbs, which leads to various complications, such as pressure sores, hypostatic pneumonia, joint stiffness and deep vein thrombosis of the lower limbs; these complications 
not only affect the recovery of patients but also could lead to death[3]. The femur is the longest and heaviest tubular bone in the human body, which connects the hip joint and the knee joint. In the current environment, as the incidence of traffic accidents increases year by year, fractures caused by high-energy injuries are prone to delayed union, which causes heavy pressure and economic burden to both society and families of patients[4]. A survey has found that the incidence rate of delayed union of tubular bone fractures has been as high as $10 \%[5]$. Therefore, the research progress of analysing the regulatory factors and active mechanism of the union process of femoral shaft fractures to promote the recovery of fractures as soon as possible needs accelerating.

Parathyroid hormone (PTH) is a peptide hormone secreted by the chief cells of the parathyroid gland; it is an important regulator of the stability of the calcium and phosphorus metabolism system in the human body, which is widely used as a bone anabolic agent[6]. A study on PTH has shown that recombinant human PTH could increase the bone mineral density and bone strength, thereby greatly reducing the incidence rate of fractures[7]. PTH-related peptide (PTHrP) was first discovered as a humoral factor of malignant tumourcaused hyperlipidaemia; its amino acid N-terminal sequence is similar to PTH and could exert the same effects as PTH; therefore, it also has certain promotive effects on the union of fracture [8]. In summary, the promotive effects of PTHrP on the union of fracture were further analysed based on the mouse femoral shaft fracture models to provide a reliable reference for clinical application.

\section{MATERIALS AND METHODS}

\section{Animals and grouping:}

Specific pathogen free mice with PTHrP gene knockout were selected as the test system and were kept in an environment of temperature at $22 \sim 26^{\circ}$ and a humidity of $50 \sim 70 \%$, and the artificial illumination lasted for $12 \mathrm{~h}$ per day. The PTHrP gene knockout heterozygous $(\mathrm{PTHr}+/-)$ mice, male and female, were mated in same cages for the reproduction of the progeny mice. Based on Mendel's laws of inheritance, the new-born mice would be in 3 genotypes, the wild-type (WT), the homozygous (PTHrP-/-) and the heterozygous $(\mathrm{PTHrP}+/-)$. However, since the homozygous mice died in the early stages of embryos, these could not be obtained for experiments. Thus, a total of 32 littermate WT and heterozygous (PTHrP+/-) mice were obtained by genotype identification. The mice were caged after 4-w old and were normally reared till 8-w old.

\section{Model construction and grouping of mouse femoral shaft fracture:}

First, each experimental mouse was weighed, anesthetized with an injection of $10 \mu \mathrm{l} / \mathrm{g}$ of $3.5 \%$ chloral hydrate into the abdominal cavity. After the mouse was successfully anesthetized, skin of the surgical area was prepared with ophthalmic scissors and skin preparation blades and the middle parts of the hind limb femurs of the mice were disinfected. The sterile operation fabric and the sterile hole towel were draped. Then, a $5 \sim 8 \mathrm{~mm}$ incision was made with a scalpel, the skin layers were cut open successively, the muscles were separated and the middle part of the femur was exposed. A $0.5 \mathrm{~mm}$ intramedullary nail was inserted along the upper edge of the patellar tendon until it reached the middle of the femoral shaft as an intramedullary fixation device. Next, the femur lateral muscle gap of the mouse was bluntly separated to the femur and the middle part of the femur was laterally cut with the tip of a pair of long-toothed scissors, the intramedullary nail for internal fixation was avoided. Once the femoral shaft fracture of the mouse was confirmed, the incisions were sutured[9].

After the fracture model was constructed, the mouse was placed in a clean mouse cage for recovery. All mice were randomly divided into 4 groups, the WT control group, the $\mathrm{PTHrP}+/-$ control group, the WT treated group and the $\mathrm{PTHr}+/$ - treated group with 8 mice in each group. Mice in both treated groups were administered with $80 \mu \mathrm{g} / \mathrm{kg} / \mathrm{d}$ of PTHrP (1-34) subcutaneously and mice in both control groups were administered saline subcutaneously each day.

\section{Detection index and specimen preparation:}

Effects of PTHrP on the extent of union of femoral shaft fracture in mice was observed after $2 \mathrm{w}$ of fracture. The mice were executed, bilateral femurs of mice in each group were fixed with $75 \%$ alcohol for X-ray examination (22 kV for 4 min exposure) and Micro-CT scans $(98 \mathrm{~mA}, 100 \mathrm{kV}$, the resolution was $18.2 \mu \mathrm{m}$, and the single rotation angle was $0.9^{\circ}$ ). Effects of PTHrP on porosis of femoral shaft fractures in mice was observed $2 \mathrm{w}$ of introducing fracture. The callus tissues of mice were paraffin-embedded, sectioned, and observed after being stained. The paraffin sections were prepared by placing the samples in an Eppendorf tube, decalcification solution was added and allowed to stand for $20 \mathrm{~d}$ to complete the process of decalcification. The 
bone tissues were successively soaked in PBS with $15 \%$ sucrose and $7.5 \%$ glycerol, PBS with $15 \%$ sucrose, and PBS with $7.5 \%$ glycerol for $12 \mathrm{~h}$ in each solution, and dehydrated in gradient alcohol. The tissues were then soaked in conventional xylene and embedded in paraffin. The paraffin block was sectioned to obtain $5 \mu \mathrm{m}$ thick sections, the sections were attached to a glass slides and baked in a baking machine. The sections were then dewaxed by placing in xylene for $5 \mathrm{~min}$ each time for 3 times until the sections were transparent. The remaining xylene on the sections was rinsed, dried and placed in $100 \%$ alcohol for 5 min twice. The sections were then liquefied with a successive gradient of 95 , 80 , and $70 \%$ alcohol for $3 \mathrm{~min}$ in each gradient, and were finally rinsed in running water for $10 \mathrm{~min}$. The sections were stained with haematoxylin for $10 \mathrm{~min}$, rinsed in running water for $5 \mathrm{~min}$, differentiated in $1 \%$ hydrochloric acid for $10 \mathrm{~s}$, rinsed in running water until the nuclei became blue under an optical microscope. The sections were then stained with Eosin for $2 \mathrm{~min}$, dehydrated with 80,95 , and $100 \%$ gradient alcohol for $2 \mathrm{~min}$ in each gradient. The sections were processed with xylene twice until they were transparent, for $5 \mathrm{~min}$ each time and were submitted to image collection after being sealed with neutral gum.

Effects of PTHrP on the formation of osteoblasts after femoral shaft fracture in mice was observed after HE staining, total collagen staining, and collagen I immunohistochemical staining. The formation of osteoblasts at the fracture sites after femoral shaft fracture of mice in each group were analysed and compared. Collagen I immunohistochemical staining was performed by dewaxing and dehydrating the sections by xylene followed by liquefied in gradient alcohol and rinsed in running water for $5 \mathrm{~min}$. Sections were then incubated for 2 hat room temperature in supersaturated picric acid straight red staining solution. The sections were then rinsed in running water for $5 \mathrm{~min}$, counterstained with haematoxylin for $5 \mathrm{~min}$, rinsed in running water for $3 \mathrm{~min}$, differentiated with $1 \%$ hydrochloric acid alcohol for $3 \mathrm{sec}$, rinsed in running water until the nuclei became blue under optical microscope. Sections were then dehydrated with gradient alcohol, processed till transparent with xylene, sealed with neutral gum, and submitted for image collection.

\section{Mechanism of action of PTHrP signalling pathway in the union of fracture:}

At present, it was not confirmed that by administering a small dose of PTHrP to the animal fracture model, the amount of callus formation at the fracture ends can be increased, the mechanical strength is improved, and union of fracture is accelerated. Through analysis, it is believed that PTH is an important hormone that maintains the balance of calcium and phosphorus metabolism in vivo; thereby maintaining the balance of blood calcium in the body. PTH is a single-strand polypeptide chain molecule containing 84 amino acid residues, and the biological activity of PTHrP is similar to that of PTH. At present, the mechanism by which $\mathrm{PTH}$ promotes fracture union is not clear. It is thought that PTH effect is mediated by G protein-coupled receptor (PTHR1), which activates adenylate cyclase after PTH binding and then activates protein kinase A(PKA). When PKA is activated, it causes changes in the expressions of cyclin and related kinase inhibition agents. This pathway can directly stimulate the survival signal of osteoblasts, reduce the apoptosis of osteoblasts, thereby increasing the number of osteoblasts; at the same time, it can affect certain bone factors and cytokines by regulating these factors, which intermittently promotes the proliferation and differentiation of osteoblasts.

Many researchers have used the embryonic stem cell homologous gene recombination technology to breed PTHrP knockout mice; however, PTHrP homozygous mice would die after births due to asphyxia and their chondrodysplasia is obvious, indicating that the PTHrP gene can stimulate chondrocyte proliferation during embryonic development and the effect is significant. After the progeny mice were born and survived, the main role of PTHrP was to promote the bone formation of osteoblasts. However the PTHrP knockout mice show osteoporosis due to defect in bone formation. Therefore, it appears that PTHrP can exert an antiosteoporosis effect by promoting the proliferation of osteoblasts.

During the development of bone, the PTH/PTHrP signaling pathway is in a mutually regulated relationship with the hedgehog $(\mathrm{Hh})$ signalling pathway. The $\mathrm{Hh}$ signalling pathway is a key regulatory pathway in embryonic development that controls the colonization of cells. The union of fractures includes intramembranous ossification and endochondral ossification. Although the main process of fracture union is different from that of embryonic bone, they are essentially the same. Both processes are regulated by the same molecular mechanism. Therefore, it is considered that the fracture union is the process of re-emergence of bone development. In vertebrates, there are 3 downstream transcription factor GLI proteins, GLI1, GLI2, and GLI3. Activation of the Hh signalling pathway promotes 
GLI1 expression and inhibits GLI3 expression. It has been found that the $\mathrm{Hh}$ signalling pathway plays an important role in the union process of fractures. The Indian hedgehog (Ihh) is mainly expressed in cartilage calluses, which plays an important role in regulating the proliferation and differentiation of chondrocytes and osteoblasts. It not only works in the early stage of fracture union but also increases calcium deposition, thereby increasing the bone mineral density.

\section{Statistics analysis:}

SPSS 20.0 statistics software was used for data analysis. The measurement data were expressed as mean \pm standard deviation and were submitted to the homogeneity test of variance. $\mathrm{P}>0.05$ indicated the homogeneity of variance, and the pair comparisons between each mean number were analysed by one-way ANOVA. $\mathrm{P}<0.05$ indicated inhomogeneity of variance, and the one-way ANOVA should be corrected by Welch. The pair comparisons of multivariate analysis were performed by the least significant difference method. $\mathrm{P}<0.05$ indicated the statistical significance of the difference.

\section{RESULTS AND DISCUSSION}

Mice after PTHrP gene knockout were divided into 2 genotypes, i.e. the WT and PTHrP+/-. Fig. 1 showed the results of X-ray examination after $2 \mathrm{w}$ of saline treatment and PTHrP administration in both genotype mice and fig. 2 showed the results of micro-CT scans. Based on the imaging results, the analysis of the fracture sites of mice in each group revealed that after $2 \mathrm{w}$ of fracture, among the saline-treated mice in the control groups, compared to the WT control group, the fracture lines of mice in the $\mathrm{PTHrP}+/$ - group were clearly visible, the fracture ends of mice in the $\mathrm{PTHrP}+/$ - group were not tightly connected, with gaps between the ends, and the callus volumes of mice in the $\mathrm{PTHr}+/$ - group were significantly smaller than those in the WT group $(\mathrm{p}<0.05)$. Meanwhile, the bone mineral density of callus sites of mice in the PTHrP+/group was lower than that in the WT group $(\mathrm{p}<0.05)$. After $2 \mathrm{w}$ of fracture, among mice administrated with PTHrP in the treated groups, the fracture lines of mice in the $\mathrm{PTHrP}+/$ - administration group were blurred, the fracture ends of mice in the $\mathrm{PTHrP}+/$ - treated group were tightly connected and a large number of osseous calluses had formed; in addition, the density of these calluses was higher and the overall condition of fracture union was not significantly different from that of the WT group, which was not statistically significant. After $2 \mathrm{w}$ of fracture, in terms of the comparison between mice in the WT control group and the WT treated group, compared to the saline-treated mice, the fracture lines of the PTHrP-treated mice were basically disappeared, the callus volumes of the PTHrP-treated mice were decreased; however, the bone mineral density of the calluses was increased $(p<0.05)$, and the overall fracture union condition of the PTHrP-treated mice was the optimal. After $2 \mathrm{w}$ of fracture, in terms of the comparison between mice in the $\mathrm{PTHrP}+/$ - control group and the $\mathrm{PTHrP}+/$--treated group, compared with the saline-treated mice, the fracture lines of the PTHrP-treated mice basically disappeared, the callus volumes at the fracture sites of the PTHrP-treated mice were increased and the bone mineral density of the calluses was increased, indicating the overall fracture union condition of the PTHrP-treated mice was better than that of the saline-treated PTHrP $+/$ - mice $(\mathrm{p}<0.05)$. The results of this experiment indicated that the loss of endogenous PTHrP affected the progress of fracture union, leading to the delayed fracture union; after PTHrP administration intervention, the delayed union due to PTHrP deficiency could be improved, and the fracture union in WT mice was also enhanced.

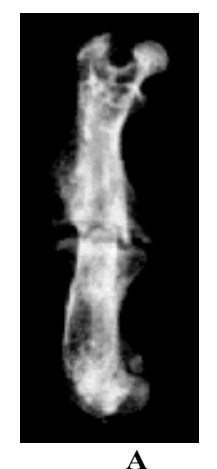

Fig. 1: X-ray examination results A: The WT group, B: the PTHrP $\mathrm{P}^{+/}$group, C: the WT treated group, D: the $\mathrm{PTHrP}^{+/-}$treated group 27
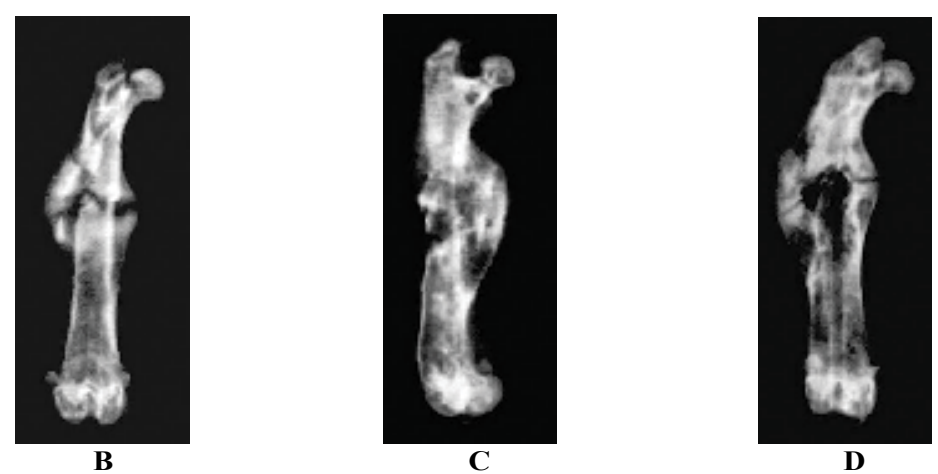

D 

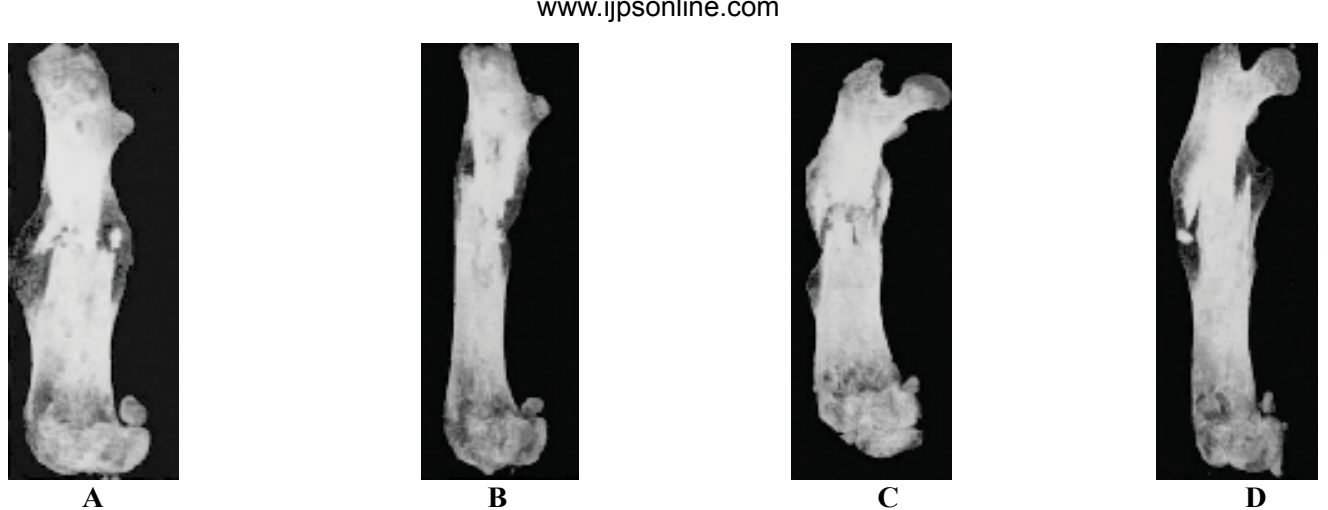

Fig. 2: The comparison of Micro-CT scan results

A: the WT group, B: the PTHrP ${ }^{+/}$group, C: the WT treated group, D: the $\mathrm{PTHrP}^{+/-}$-treated group

The bone tissues of mice in each group were paraffin embedded, sectioned and submitted to histological staining; the results of HE staining were used to analyze the formation of calluses and were calculated through computer software. The results showed that after $2 \mathrm{w}$ of fracture, among the saline-treated mice in the control groups, compared to the WT control group, the total callus area of mice in the $\mathrm{PTHrP}+/$ group was reduced by $29.8 \%$, the osseous callus area of mice in the $\mathrm{PTHrP}+/$ - group was decreased by $34.6 \%$, and the differences were statistically significant $(\mathrm{p}<0.05)$, however, the cartilage callus area of mice in the $\mathrm{PTHrP}+/$ - group was increased by $97 \%$, compared to the WT control group, the difference was statistically significant $(\mathrm{p}<0.05)$. After $2 \mathrm{w}$ of fracture, in terms of the comparison between mice in the $\mathrm{PTHrP}+/$ - control group and the $\mathrm{PTHr}+/--$ treated group, compared with the saline-treated mice, the total callus area and the osseous callus area of the PTHrP-administrated mice were obviously increased, and the cartilage callus area was decreased $(\mathrm{p}<0.05)$. After $2 \mathrm{w}$ of fracture, in terms of the comparison between mice in the WT control group and the WT treated group, compared to the saline-treated mice, the osseous callus area of the PTHrP-administrated mice increased by $81.6 \%$, the cartilage callus area was decreased, and the differences were statistically significant $(p<0.05)$. Fig. 3 showed the results of HE staining of callus tissues after $2 \mathrm{w}$ of saline treatment and PTHrP administration intervention in $\mathrm{WT}$ and $\mathrm{PTHrP}+/-$ mice.

The results of this study indicated that endogenous PTHrP deficiency inhibited the formation of callus and delayed the rate at which cartilage calluses were converted into osseous calluses, leading to delayed fracture union. After PTHrP administration, the formation of callus was promoted, the rate of conversion of cartilage callus into osseous callus was also enhanced.
The total collagen staining and collagen I immunohistochemical staining of mice callus tissues in each group were used to compare and analyse the changes in the formation of osteoblasts in the callus formation sites of mice. The analysis of the staining results revealed that after $2 \mathrm{w}$ of fracture, the number of osteoblasts in the PTHrP+/- group was significantly reduced compared to the WT group; in addition, \% collagen-positive area of mice in the $\mathrm{PTHrP}+/$ - control group was significantly lower than that in the WT control group $(\mathrm{p}<0.05)$. In mice with the same genotype, compared to the saline-treated group, the number of osteoblasts in the PTHrP treated group and \% positive area of collagen I increased significantly $(p<0.05)$. Fig. 4 showed the results of HE staining of callus tissues after $3 \mathrm{w}$ of saline treatment or PTHrP treatment in WT and $\mathrm{PTHrP}+/$ - mice.

The results of this study indicated that endogenous PTHrP deletion inhibited the formation and proliferation of osteoblasts and reduced the production of collagen I. However, after PTHrP administration, the proliferation and activation of osteoblasts were stimulated and the bone matrix protein synthesis was promoted. Therefore, by promoting the bone formation of osteoblasts and deepening the formation and shaping of calluses, the union of fracture was promoted.

In general, the non-union of the fracture in the clinical field refers to that 3 mo after the normal treatment of the fracture, the X-ray examination indicated that the bone connection at both ends of the fracture site is not obvious and the failure to reach the union standard is called delayed fracture union. The delayed union of fracture is a common complication of fractures. Delayed union of fractures in one site may be related to a variety of factors, including self-factors, type of fracture, treatment plans, fixation conditions, and presence or absence of infection. 

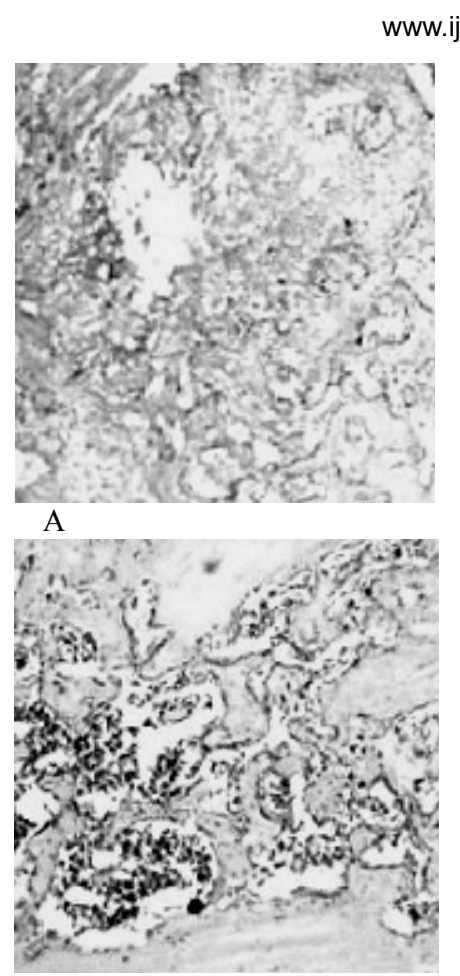

C

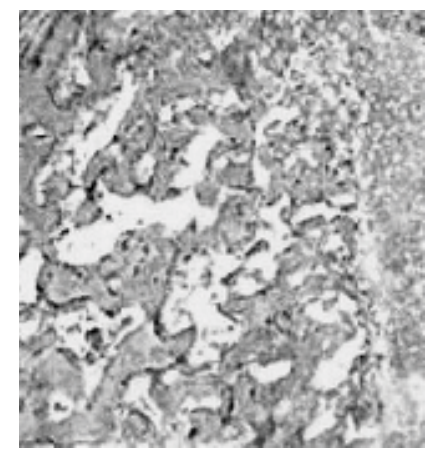

B

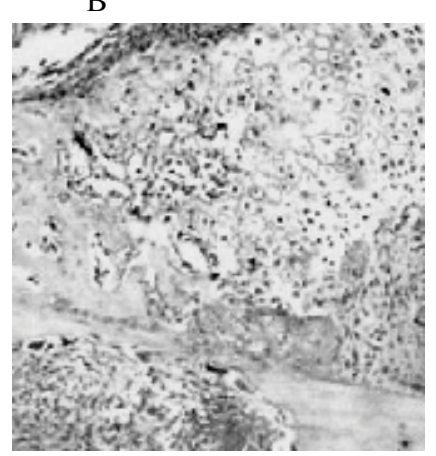

$\mathrm{D}$

Fig. 3: HE staining results of callus tissues

A: the WT group, B: the PTHrP $\mathrm{P}^{+/}$group, C: the WT treated group, D: the $\mathrm{PTHrP}^{+/-}$-treated group

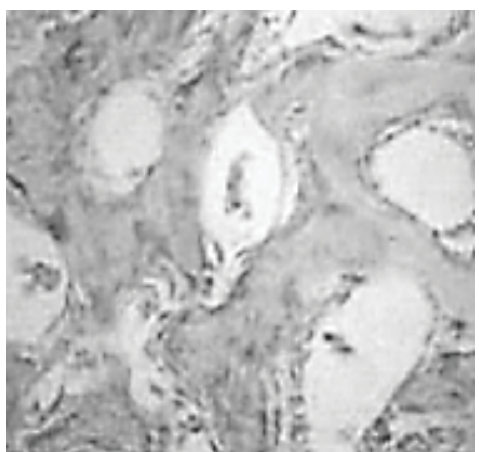

A

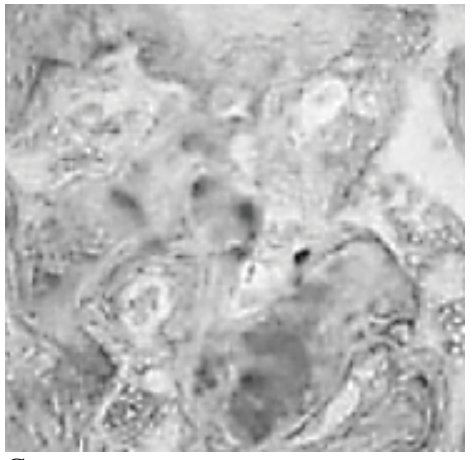

$\mathrm{C}$

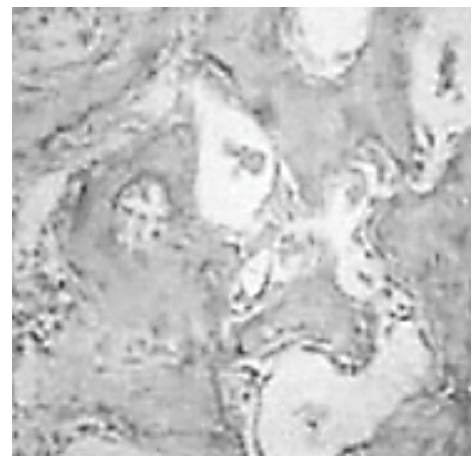

B

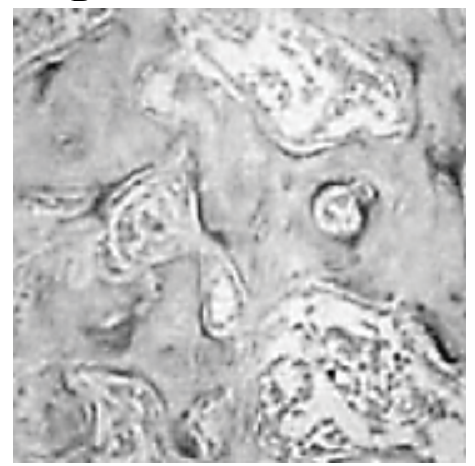

Fig. 4: Immunohistochemical staining results of callus tissue collagen I

A: the WT group, B: the PTHrP ${ }^{+/}$group, C: the WT treated group, D: the $\mathrm{PTHrP}^{+/-}$treated group

Delayed union of femoral shaft fracture is common in all types of delayed fracture union, and is mainly related to the following 4 factors, the physiological factor, after the fracture occurs, the nutritional artery at the fracture site was either damaged or cut, resulting in loss of influences of local blood circulation; in addition, after the femoral shaft fracture, due to the increased blood loss, the blood supply to the backbone and periosteum would be affected, which could lead to delayed fracture union. The self-factor which is related to unreasonable functional exercise after fracture and the premature weight bearing will cause internal 
fixation failure, resulting in delayed fracture union. The iatrogenic factor, an improper location and extent of fracture fixation, the unreasonable application of fixed materials and so on causing stress interference to the fracture site, leading to delayed fracture union; in addition, the improper treatment of open fractures may lead to infection, which may cause the formation of granulation tissue, abnormal activities of fracture ends, etc., and also affects the bone union; and finally the stress factor, if the bone stress is too small, the mechanical induction of tissue differentiation will be lowered, which is easy to cause delayed fracture union; besides, if the stress is too large, the living bone will have reactive surface absorption, which will easily lead to bone atrophy.

The femur is the longest tubular bone in the human body, which connects the hip joint and the knee joint, with a slight forward curvature. With the rapid development of transportation in recent years, the incidence rate of femoral shaft fractures is significantly higher than before and the femoral shaft fractures are mostly highenergy trauma, which is extremely harmful to patients. The union of the fracture is a long, complicated, and continuous process, especially after the femoral shaft fracture, the incidence rate of delayed fracture union is also increasing year by year due to various factors. Clinically, there are various ways to treat the delayed fracture union, including bone grafting, high-energy shock wave, electrical stimulation therapy, and internal fixation. However, the effect is not satisfactory, and $10 \%$ of the patients still have delayed fracture union. In addition, the resulted series of complications have also caused harm to patients, which has brought enormous burdens to families and society.

At present, there are many studies on the treatment methods of delayed fracture union, but the mechanism and regulation mechanism for delayed union is relatively rare. PTHrP is a humoral factor in the human body and can be expressed in tissues such as bone, thyroid, and cardiovascular tissues. Studies reported abroad have confirmed that endogenous PTHrP promotes bone formation. In vitro experiments have also found that PTHrP can accelerate the bone formation and promote fracture union. Therefore, the role of PTHrP in promoting fracture union and related mechanisms were analysed from the perspective of genetics in the mouse femoral shaft fracture models. Mice after PTHrP gene knockout were divided into 2 genotypes, i.e. the WT and $\mathrm{PTHrP}+/-$, which were respectively treated with saline and PTHrP (1-34) interventions to analyse the functions of PTHrP in the union of the fracture. It was found that PTHrP (1-34) administration could accelerate the formation of osteoblasts, increase the bone strength, and promote the fracture union. It was also found that the formation of osteoblasts and calluses were inhibited in mice with PTHrP gene deletion after fracture, which could lead to the delayed fracture union. Therefore, $\mathrm{PTHrP}$ has great application prospects as a therapeutic drug for fracture union. In the subsequent studies, the clinical efficacy after drug application can be taken as the focus of observation.

\section{REFERENCES}

1. Singh R, Rohilla R, Gawande J. To evaluate the role of platelet-rich plasma in healing of acute diaphyseal fractures of the femur. Chin J Traumatol 2017;20(1):39-44.

2. Yin $Z$ Y, Yin J, Huo Y F. Rapamycin facilitates fracture healing through inducing cell autophagy and suppressing cell apoptosis in bone tissues. Eur Rev Med Pharmacol Sci 2017;21(21):4989-98.

3. Miura T, Kijima H, Ishikawa N. Comparison of Atypical and Osteoporotic Femoral Shaft Fractures in the Elderly: A Multicenter Study. Adv Orthop 2018;1-5.

4. Zhang R, Yin Y, Li S. Traction table versus double reverse traction repositor in the treatment of femoral shaft fractures. Sci Rep 2018;8(1):5952.

5. Kim M B, Cho J W, Lee Y H. Locking attachment plate fixation around a well-fixed stem in periprosthetic femoral shaft fractures. Arch Orthop Trauma Surg 2017;137:1-8.

6. Han L, Hu Y G, Fang W L. Inversive LISS plate in treating intertrochanteric and subtrochanteric fractures combined with femoral shaft fractures. Zhongguo Gu Shang 2017;30(7):61215.

7. Giordano V, Paes R P, De-Queiroz G B. What is the ideal working length for bridge plating osteosynthesis of a femoral shaft fracture? A multinational online survey evaluation. Revista Do Colégio Brasileiro De Cirurgiões 2017;44(4):32839.

8. Chen A T, Morris W Z, Zirkle L G. Evaluation of Intramedullary Fixation for Pediatric Femoral Shaft Fractures in Developing Countries. J Orthop Trauma 2018;32(6):1.

9. Rajasekaran R B, Jayaramaraju D, Palanisami D R. Ipsilateral Acetabular Fracture with Displaced Femoral Head and Femoral Shaft Fracture: A Complex Floating Hip Injury. Case Reports Orthopedics 2018;1-5.

This is an open access article distributed under the terms of the Creative Commons Attribution-NonCommercial-ShareAlike 3.0 License, which allows others to remix, tweak, and build upon the work non-commercially, as long as the author is credited and the new creations are licensed under the identical terms

This article was originally published in Special
issue on "Trends in therapeutic Management of
Various Conditions" Indian J Pharm Sci 2020:82(3)
spl issue 6;xx-xx

